\title{
CONGENITAL MALARIA IN NEWBORN TWINS
}

\author{
D. A. OPARE \\ Sunyani Municipal Health Directorate, P. O Box 311, Sunyani, Ghana
}

Author for correspondence: Dr. David A. Opare

Email: oparedavid@yahoo.co.uk

Conflict of interest: None

\section{SUMMARY}

Malaria during first few months of life may be due to transplacental transfer of parasitized maternal erythrocytes. The most common clinical features of congenital malaria are fever, anaemia and splenomegaly. Other signs and symptoms include hepatosplenomegaly, jaundice, regurgitation, loose stools, and poor feeding. A 28 year old woman (G2P1A), with 36 weeks gestation, reported to a health facility in Sunyani on $22^{\text {nd }}$ February 2009 with history of labour pains, without fever. According to the mother, even though she did not sleep in insecticide treated bed net during her pregnancy, she took all the recommended drugs of sulfadoxine/pyrimethamine- intermittent preventive treatment for malaria. She delivered twins on the same day. The mother and the twins developed fever on the same day. A laboratory investigation on the three of them was positive for malaria parasites. The three were successfully treated with quinine. Congenital malaria is real and it is therefore recommended that babies born to mothers with malaria should be screened for congenital malaria.

Keywords: congenital, intermittent preventive treatment, apgar.

\section{INTRODUCTION}

Congenital malaria, defined as the presence of malaria parasites in the erythrocytes of newborns aged less than 7 days, was considered rare in endemic areas until recent studies started reporting high prevalence rates. ${ }^{1}$ Congenital malaria was first described in $1876 .{ }^{2}$ Congenital malaria can be acquired by transmission of parasites from mother to child during pregnancy or perinataly during labour. ${ }^{3}$

Congenital malaria has been documented for many years but it was previously thought to be uncommon especially in indigenous populations. ${ }^{4}$ More recent studies, however, suggest that incidence has increased and values between 0.3 to $33 \%$ have been observed from both endemic and non-endemic areas. ${ }^{4}$

Some studies have attributed the higher incidence to an increased resistance and virulence of parasite resulting from altered antigenic determinants in addition to increased reporting. ${ }^{5}$

Symptoms usually occur 10 to 30 days postpartum. ${ }^{6}$ However, the disease can be seen in a day-old baby or be delayed for weeks or months. The most common clinical features in $80 \%$ of cases are fever, anaemia and splenomegaly. $^{7}$ Other features include hepatosplenomegaly, jaundice, regurgitation, loose stools, and poor feeding. Occasionally restlessness and cyanosis can also be seen. ${ }^{7}$

Children with congenital malaria can present with fever, irritability, feeding problems, hepatosplenomegaly, anaemia and jaundice. ${ }^{8}$ Clinically, apparent congenital malaria is rare in areas in which malaria is endemic and levels of maternal antibody are high.

In Ghana, as a way of reducing malaria in pregnancy, with its consequent reduction in congenital malaria, all asymptomatic pregnant women receive regular doses of sulphadoxine/pyrimethamine as an intermittent preventive treatment (IPT) during the second and third trimesters of pregnancy. The first dose (IPT1) is given after 16weeks gestation, the second dose (IPT2) is given at least one month after the first dose and the third dose (IPT3) is given at least one month after the second dose. ${ }^{9}$

\section{Case Report}

A 28 year old woman $\left(\mathrm{G}^{2} \mathrm{P}^{1 \mathrm{~A}}\right)$, with 36 weeks gestation, reported to a Health Facility in Sunyani on $22^{\text {nd }}$ February 2009 at $8.30 \mathrm{pm}$ with history of labour pains, without fever. Though the mother had no fever on admission, she developed fever of $38.5^{0} \mathrm{C}$ with rigor during the course of her labour on the second day.

According to the mother, even though she did not sleep in insecticide treated bed net during her pregnancy, she took all the recommended doses of sulfadoxine/pyrimethamine for intermittent preventive treatment for malaria (IPT1, IPT2 and IPT3). She delivered identical normal healthy male twins on $23^{\text {rd }}$ February 2009 at $1.45 \mathrm{pm}$ through spontaneous vaginal delivery. 
The first twin had a birth weight of 2.8kilograms and apgar score of $8 / 10$ and $10 / 10$ in $1^{\text {st }}$ and $5^{\text {th }}$ minutes respectively.

The head circumference was $32 \mathrm{cms}$ and body length of $49 \mathrm{cms}$. The second twin had a birth weight of 2.5kilograms and apgar score of 7/10 and 9/10 for the $1^{\text {st }}$ and 5th minutes respectively. The head circumference was $31 \mathrm{cms}$ and body length was $48 \mathrm{cms}$. The mother developed fever on the day of delivery.

A blood film for malaria parasites was indicative of malaria parasites (P. falciparum). The mother had no history of having received blood transfusion.On the day of delivery, the first twin also developed fever of $38^{0} \mathrm{C}$. This fever was detected one hour after delivery. On examination, the first twin was febrile, and jaundiced but not pale. The liver and spleen were not palpable. The chest was clinically clear. The heart examination was normal.

The abdomen was soft without any distension. A blood film, taken from a thumb prick, for malaria parasites was examined and it came out to be positive for malaria parasites ( $P$. falciparum). A diagnosis of congenital malaria was made.

On the same day, 23rd February 2009, the second twin also developed fever of $38.1^{0} \mathrm{C}$. This fever was detected one hour after delivery. On examination, the second twin was febrile and jaundiced but not pale. The spleen and liver were not palpable. The chest was clinically clear. A blood film, taken from a thumb prick, for malaria parasites was positive (P. falciparum) and a diagnosis of congenital malaria was made.

Due to logistical constraints and lack of expertise at the laboratory, other laboratory investigations to determine other causes of the jaundice could not be done. Due to these same reasons, determining the serum bilirubin level of both twins and blood cultures to rule out bacterial infection could also not be done.

The mother was put on quinine tablets and the twins on quinine syrup. They all responded well to treatment. The fever dropped to normal on the third day for all of them and they were all discharged on the third day. A review of the twins after a week did show that the jaundice had cleared and they had no fever and was not pale. The liver and the spleen were not palpable.

The laboratory investigation for malaria parasite was negative. Similarly, the mother had no fever and the laboratory investigation was also negative for malaria.

\section{DISCUSSION}

Congenital malaria is possible if there are leakages between the mother's blood circulation and the foetus. This might have contributed to the congenital malaria, since the mother had malaria parasites in her peripheral blood. Inferring from the period that the twins had the malaria, it is unlikely that they had the malaria from a mosquito bite on the hospital ward.

In Ghana, falciparum malaria is the most common, and the incubation period is between 10 to 14days. Although logistical constraints, coupled with lack of expertise at the laboratory, did not make it possible to investigate for other causes of the jaundice in the babies, and do blood cultures. The laboratory staff have considerable expertise in reading malaria slides, which they do daily.

The twins recovered with the antimalarial drugs without any antibiotics being given, which is suggestive of the fact that they may not have any bacterial infection. The only logical conclusion is that, the twins were infected with the malaria parasites from the mother which could, at this point in time be through the maternal blood in the uterus, hence congenital.

It is worth noting that the twins also had normal birth weights. Other studies have documented conflicting birth weights. ${ }^{10}$ However, some studies have reported low birth weights in association with parasitization of the placenta. A similar association was observed in malaria endemic sub-Saharan Africa. ${ }^{11,12}$ Differences in these weights could be linked up to many factors, such as intensity of the infection, important causes of low birth weight. ${ }^{13}$

The World Health Organization (WHO) 20th Malaria Committee designated IPT as the preferred approach to reduce the adverse consequences of malaria during pregnancy. ${ }^{9}$ The mother had received prophylaxis for malaria in the form of sulphadoxine/pyrimethamine but had malaria so also the twins.

The mother of the babies attended antenatal care at a health facility in the municipality, where sulphadoxine/pyrimethamine is given to mothers to swallow under direct observation by the midwives. The mother took three doses at the required and acceptable interval and it was recorded in her antenatal record card.

In view of this, there is the need to investigate further the possibility of resistance of the parasite to the drug in Ghana. Furthermore, the need to research into the prevalence of congenital malaria in Ghana to inform policy can be discussed. 


\section{CONCLUSION}

Although malaria continues to be a major public health importance in Ghana and pregnant women and children have been described as vulnerable groups, there have been few epidemiological studies to determine congenital malaria.

Congenital malaria is real and it is therefore recommended that babies born to mothers with malaria should be screened for congenital malaria. Furthermore all neonates with unexplained fever should be evaluated for congenital malaria and treated with effective anti-malaria drugs.

\section{ACKNOWLEDGMENTS}

I wish to thank Dr. Ivy Frances Osei for her contribution in offering critical comments in this case report. I am grateful to the patient for responding to the numerous questions I asked her. The assistance of Dr F. Dery, Dr Anvoh Gervais and staff of the Maternity Unit of Sunyani Municipal Hospital, Dr Anthony Adofo Ofosu of Sene District Hospital is gratefully acknowledged.

\section{REFERENCES}

1. Sotimehina SA, Runsewe-Abioduna TI, Oladapob OT, Njokanmac OF and Olanrewajua DM. Possible Risk Factors for Congenital Malaria at a Tertiary Care Hospital in Sagamu, Ogun State, SouthWest Nigeria Journal of Tropical Pediatrics 2008; 54(5):313-320

2. Romand S, Bourée P, Gelez J , Bader-Meunier B, Bisaro F, Dommergues JP, 1994. Congenital malaria. A case observed in twins born to an asymptomatic mother. Journal: Presse médicale (Paris, France: 1983) (Presse Med) published in France. (Language: Fre). 1994 Apr 30-May 7; 23 (17):797-800
3. Menendez C. Malaria during pregnancy: A prior ity area of malaria research and control Parasitol Today 1995;11:178-183.

4. Fischer PR. Malaria and Newborn J Trop Pediatr 2003; 49:132-134.

5. Mukhtar MY, Lesi FEA, Iroha EU, Egri-Okwaji MTC, Mafe AG. Congenital malaria among inborn babies at a tertiary centre in Lagos, Nigeria $J$ Trop Pediatr 2006; 52(1):19-23.

6. Behrman RE, Keligman R, Jenson HB. Nelson Textbook of Pediatrics 17th ed. Philadelphia: Saunders 2004: p1140

7. Remington JS, Klein JO. Infectious Diseases of the Fetus and Newborn Infant 3rd ed. Philadelphia: Saunders 1995 p765 - 768

8. Basipinar O, Bayraktaroglu Z, Karsligil T, Bavram A, Coskun Y. A rare cause of anemia and thrombocytopenia in a newborn: congenital malaria Turk J Pediatr 2006;48:63-5.

9. GHS/NMCP/Global Fund/JHPIEGO. Intermittent Preventive Treatment (IPT) of malaria in pregnancy. May 2005. p29-31

10. McGregor IA, Wilson ME, Dillewicz WZ. Malaria infection of the placenta in the Gambia,West Africa:its incidence and relationship to still births, birth weight and placenta weight Trans $R$ Soc Trop Med Hyg 1983;77:232

11. Steketee RW, Nahlen BL, Parise ME, Menendez $\mathrm{C}$. The burden of malaria in pregnancy in malariaendemic areas Am J Trop Med Hyg 2001;64:28-35

12. Menendez C, Ordi J, Ismail M, Ventra P, Aponte J, Kahigwa E, Font F, Alonso P The impact of placenta malaria on gestational age and birth weight. J Inf Dis 2000; 181:1740-1745

13. Lawoyin TO. Maternal weight and weight gain in Africans: Its relationship to birth weight. J Trop Pediatr 1991; 37:166-171 